\title{
Swimming characteristics of gyrotactic microorganisms in low-Reynolds-number flow: Chlamydomonas reinhardtii
}

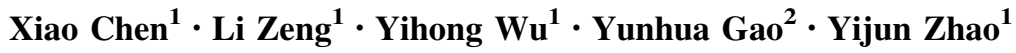 \\ ${ }^{1}$ State Key Laboratory of Simulation and Regulation of Water Cycle in River Basin, China Institute of Water Resources and Hydropower \\ Research, Beijing 100038, China \\ 2 National Institute of Metrology, Beijing 100013, China
}

Received: 28 April 2017/Revised: 18 July 2017/Accepted: 19 July 2017/Published online: 1 August 2017

(c) Joint Center on Global Change and Earth System Science of the University of Maryland and Beijing Normal University and Springer-Verlag GmbH Germany 2017

\begin{abstract}
The swimming characteristics of gyrotactic microorganisms are significant to understand the ecological activities in lakes, rivers and oceans. The swimming velocity of a typical motile microorganism, Chlamydomonas reinhardtii, was measured for both still water and low-Reynolds-number flow, based on a microfluidic system. Results show that the swimming speed is subject to Gaussian distribution for the still water, and corresponding mean swimming speed is $41 \mu \mathrm{m} / \mathrm{s}$. The streamwise mean swimming velocity, $35 \mu \mathrm{m} / \mathrm{s}$, in the moving water is slightly less than that in the still water. It is also shown that the swimming direction in the horizontal plane is dominated by cell randomness for the still water, and $80 \%$ of the cells are aligned with the ambient flow when the flow velocity exceeds $333 \mu \mathrm{m} / \mathrm{s}$. The standard deviation of swimming direction and the percent of swimming direction in the streamwise direction can reach a stable status with the increase of the flow velocity.
\end{abstract}

Keywords Gyrotactic microorganisms · Low-Reynoldsnumber flow $\cdot$ Motile cells $\cdot$ Swimming velocity

\section{Introduction}

Phytoplankton, as the primary producers of aquatic ecosystems, play a significant role in the material and energy cycles (Heisler et al. 2008; Sengupta et al. 2017). The swimming behavior of phytoplankton species is essential to various ecological phenomena, including the

Li Zeng

lizeng@iwhr.com formation of bioconvection (Pedley et al. 1988; Hwang and Pedley 2014a, b), microscale patches (Durham et al. 2013; Goldstein 2015), phytoplankton thin layers (Durham et al. 2009), harmful algal blooms (HABs) (Durham and Stocker 2012), etc.

Regarding the swimming characteristics of motile phytoplankton, there have been various theoretical, numerical and experimental studies (Durham et al. 2009, 2013; Pedley et al. 1988; Pedley and Kessler 1990; Son et al. 2015), with focus on the swimming behaviors of Chlamydomonas reinhardtii (Martin et al. 2016; Sineshchekov et al. 2000), Chlamydomonas nivalis (Hill and Häder 1997), Heterosigma akashiwo (Durham et al. 2009, 2013) and Volvox (Drescher et al. 2009; Goldstein 2015). Two types of models, i.e. continuum model (Pedley et al. 1988; Pedley and Kessler 1990) and individual model (De Lillo et al. 2014; Durham et al. 2011) are used to describe the swimming behaviors, which are dependent on the intrinsic characteristics of cells and external conditions (light intensity, the vorticity of ambient flow, etc.).

With regard to the swimming characteristic of algal cells, lots of efforts have been made for the still water. The swimming velocity and trajectories were measured, mainly based on the microscope-based (Häder and Lebert 1985; Hill and Häder 1997) and the laser-based tracking methods (Vladimirov et al. 2000, 2004). The microscope-based tracking method can provide a high spatial resolution, while the laser-based tracking method can offer a large scope. Häder and Lebert (1985) investigated the positive phototaxis and negative phototaxis of Euglena gracilis under various light sources. Hill and Häder (1997) investigated the random walk model of swimming microorganisms. Vladimirov et al. (2004) measured the velocity 
distributions in populations of $C$. nivalis, using the laserbased tracking method.

There have been various studies on the response of motile microorganisms to flow. Kessler (1985) pointed out that the aggregation of gyrotactic cells was dependent on the flow shear. Marcos and Stocker (2006) studied the effects of vortices on the distribution of microorganisms in a microchannel. Durham et al. $(2011,2013)$ studied the distribution of motile cells in a steady vortex and the formation of phytoplankton layer triggered by shear. Croze et al. (2013) investigated the dispersion of motile microorganisms in laminar and turbulent channel flows. Lillo et al. (2014) reported the influences of acceleration of flow on the formation of algal patches in a rotating cylindrical tube. Martin et al. (2016) observed the focusing of $C$. reinhardtii in the flow through a microchannel under the combined action of vorticity and phototaxis. Rusconi et al. (2014) found that the flow shear hampered the chemotaxis of Bacillus subtilis.

This work is to understand the swimming characteristics of one typical gyrotactic microorganism, C. reinhardtii, in the low-Reynolds-number flow, based on the microscope tracking method. The specific objects are: (1) to measure the swimming velocity and direction in the still water and low-Reynolds-number laminar flow, and (2) to examine the effects of flow on the swimming velocity of algal cells.

\section{Materials and methods}

\subsection{Experimental setup}

The experimental system consists of a microfluidic system, an imaging system and a microfluidic chip, as shown in Fig. 1. The microfluidic system contains microfluidic flow control system (MFCS-EZ, Fluigent Company) and flowrate platform (FRP, Fluigent Company). The imaging system includes microscope (IX83, Olympus), camera (DP80, Olympus) and imaging analysis software (CellSens, Olympus). The microfluidic chip is made of PDMS and a standard glass slide. The main microchannel in the chip has a length of $58,500 \mu \mathrm{m}$, a width of $500 \mu \mathrm{m}$ and a depth of $50 \mu \mathrm{m}$. A $120-\mu \mathrm{m}$-wide and $7500-\mu \mathrm{m}$-long channel is connected to the main channel to check the flow pattern in the main channel. The diagram of experimental setup and the enlarged view of the microfluidic are shown in Fig. 1.

Fig. 1 Experimental sketch

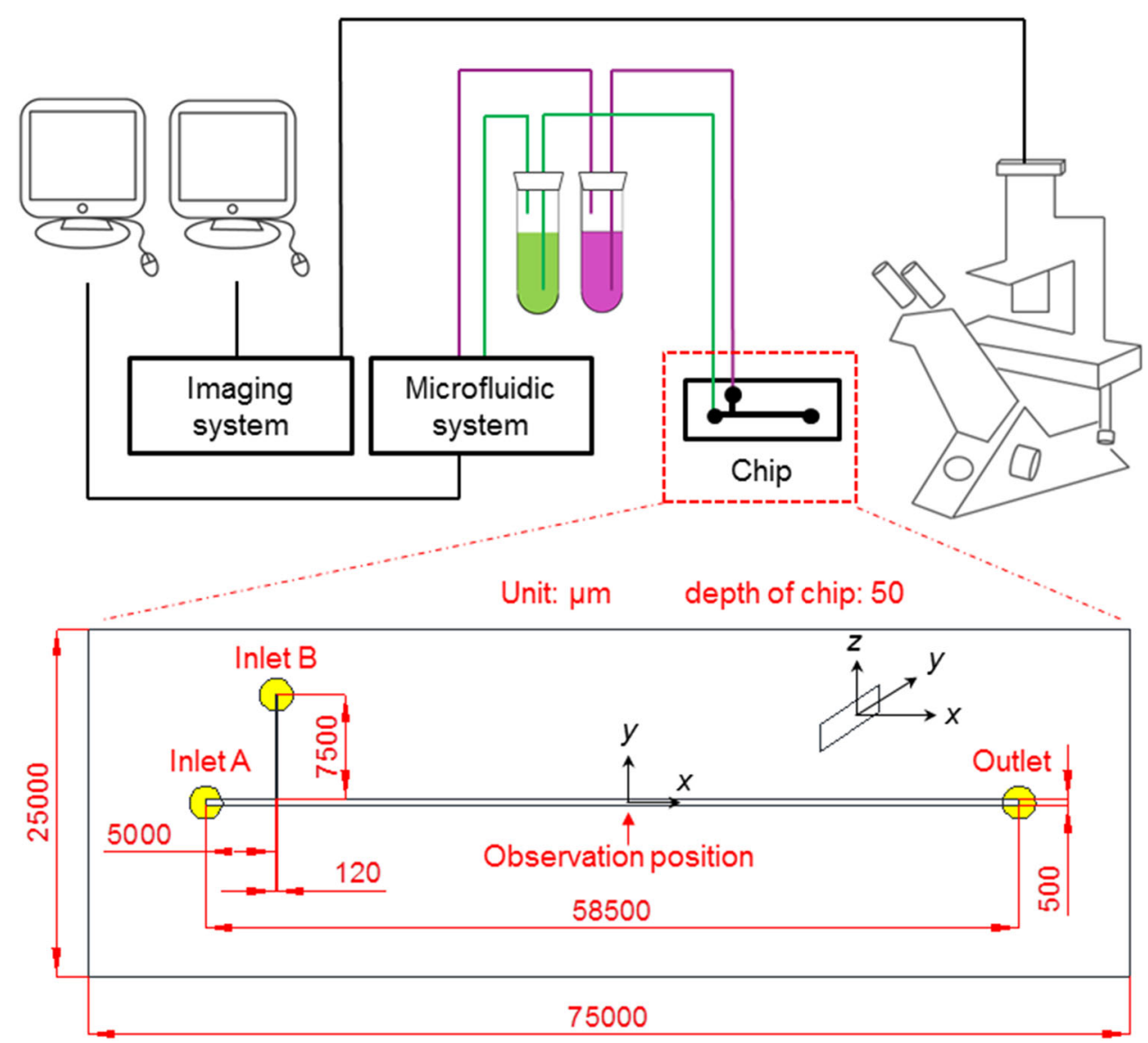




\subsection{Experimental materials}

The experimental materials include algal cells $(C$. reinhardtii, Institute of Hydrobiology, Chinese Academy of Sciences), ultrapure water and tracers (potassium permanganate). The algal cells were incubated at $25^{\circ} \mathrm{C}$ in selenite enrichment (SE) medium, and the daily cycle was $12 \mathrm{~h}$ of white light (2000 lux) and $12 \mathrm{~h}$ in the dark. The cells used for experiments were taken from the upper layer of suspensions which were vibrated for $5 \mathrm{~min}$ and stood for $30 \mathrm{~min}$. The tracers were injected from the Inlet B to observe the flow pattern through the main channel, and the ultrapure water and algal cells were injected from Inlet A.

\subsection{Statistical analysis}

The velocity of cell migration $\left(U_{x}, U_{y}\right)$ is calculated by

$U_{x}=k_{1} \frac{x_{i}-x_{i-1}}{\Delta t}$

$U_{y}=k_{2} \frac{y_{i}-y_{i-1}}{\Delta t}$

where $\left(x_{i}, y_{i}\right)$ and $\left(x_{i-1}, y_{i-1}\right)$ are the pixel positions of algal cells in the $i$-th frame and $i-1$-th frame, $k_{1}(1.48 \mu \mathrm{m} / \mathrm{pixel})$ and $k_{2}(1.48 \mu \mathrm{m} / \mathrm{pixel})$ are the conversion coefficients between pixel and physical coordinates in the $x$ and $y$ directions, respectively, and $\Delta t(0.125 \mathrm{~s})$ is the time interval between two continuous images. The resultant speed, $U_{r}$, is defined as

$U_{r}=\sqrt{U_{x}^{2}+U_{y}^{2}}$

which represents the speed of migration of cells in the horizontal plane. For the case of quiescent water, the swimming velocities of cells $\left(V_{x}, V_{y}\right)$ are equal to the migration velocity $\left(U_{x}, U_{y}\right)$. For the moving water, the swimming velocity $\left(V_{x}, V_{y}\right)$ are given by

$V_{x}=U_{x}-u_{y z}$

$V_{y}=U_{y}$ where $u_{y z}$ is the velocity of ambient flow. The $z$-axis is perpendicular to the $x-y$ plane. The vertical position of focal plane is determined by adjusting the focal length and recording the $z$-axis step value of the microscope. $u_{y z}$ and corresponding discharge $Q$ through the cross section of the main channel are given by (Shah and London 1978; White 2005),

$$
\begin{aligned}
& u(y, z)= \frac{16 a^{2}}{\mu \pi^{3}}\left(-\frac{\mathrm{d} \hat{p}}{\mathrm{~d} x}\right) \sum_{i=1,3,5 \ldots}^{\infty}(-1)^{(i-1) / 2} \\
& \times\left[1-\frac{\cosh (i \pi z / 2 a)}{\cosh (i \pi b / 2 a)}\right] \times \frac{\cos (i \pi y / 2 a)}{i^{3}} \\
& Q=\frac{4 b a^{3}}{3 \mu}\left(-\frac{\mathrm{d} \hat{p}}{\mathrm{~d} x}\right)\left[1-\frac{192 a}{\pi^{5} b} \sum_{i=1,3,5}^{\infty} \frac{\tanh (i \pi b / 2 a)}{i^{5}}\right]
\end{aligned}
$$

where $a(250 \mu \mathrm{m})$ and $b(25 \mu \mathrm{m})$ are half-width and halfheight of the channel, respectively, $\hat{p}$ is the pressure and $\mu$ is the dynamic viscosity of water. The convergence of Eq. (6) is numerically shown very fast, and the first 10 terms $(N=10)$ of the series are qualified for a reasonable approximation to the exact solution, as shown in Fig. 2. The swimming direction is defined as the angle $(\theta)$ between the positive $y$-axis and the cell axis. $\theta$ is calculated by Eqs. (8)-(12). The positive angle represents the clockwise rotation, and the negative angle represents the counterclockwise rotation, as shown in Fig. 3.

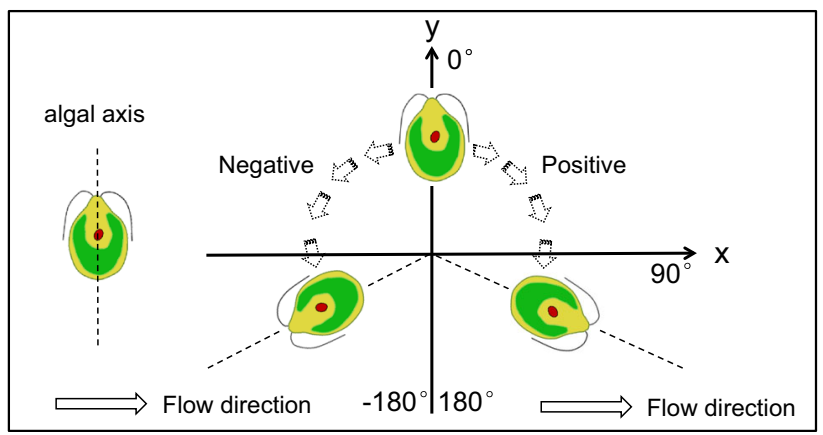

Fig. 3 Definition of swimming direction
Fig. 2 Velocity profiles for $N=5,10$ and 15 at: $\mathbf{a} y=0$ and $\mathbf{b} z=0$
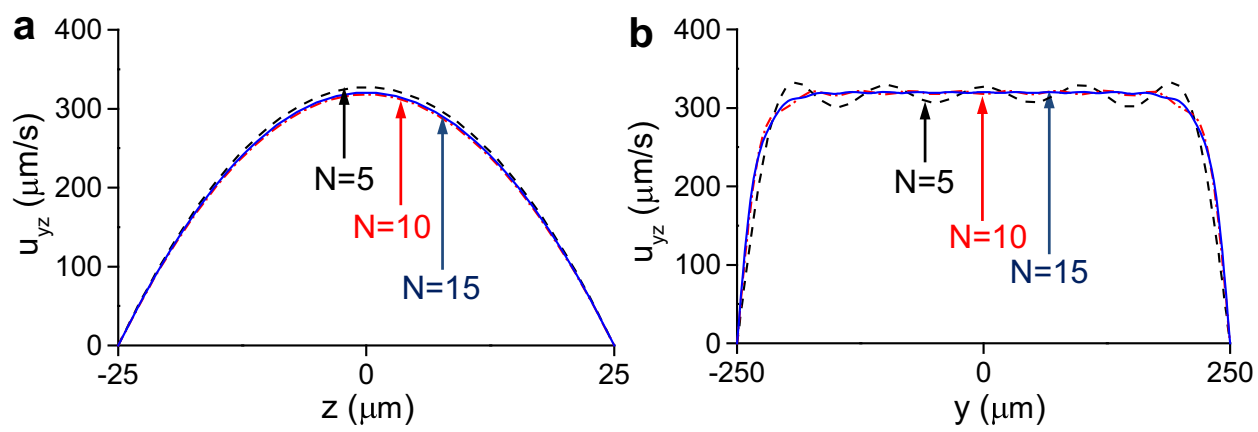
$\theta=\arctan \frac{U_{x}}{U_{y}}\left(U_{y}>0\right)$

$\theta=\pi+\arctan \frac{U_{x}}{U_{y}}\left(U_{x} \geq 0, U_{y}<0\right)$

$\theta=-\pi+\arctan \frac{U_{x}}{U_{y}}\left(U_{x}<0, U_{y}<0\right)$

$\theta=\frac{\pi}{2}\left(U_{x}>0, U_{y}=0\right)$

$\theta=-\frac{\pi}{2}\left(U_{x}<0, U_{y}=0\right)$.

\section{Results}

\subsection{Swimming characteristics in still water}

C. reinhardtii under the microscope is shown in Fig. 4. The diameter of cells ranges from 7 to $11 \mu \mathrm{m}$ in the present work. The trajectory of $C$. reinhardtii was observed at $26^{\circ} \mathrm{C}$ in a count box with a length and width of $20,000 \mu \mathrm{m}$, and a depth of $250 \mu \mathrm{m}$. The average swimming speed is approximately $41.0 \mu \mathrm{m} / \mathrm{s}$, and the maximum swimming speed can reach $160 \mu \mathrm{m} / \mathrm{s}$. The probability distribution function (PDF) of the swimming speed $V_{r}$ is approximately subject to Gaussian distribution $A_{1} /\left(\sqrt{2 \pi} \sigma_{1}\right) \mathrm{e}^{-\left(V_{r}-V_{r c}\right) /\left(2 \sigma_{1}^{2}\right)}$, where $A_{1}=0.97, \sigma_{1}=19.67$ and $V_{r c}=38.30$, as shown in Fig. 5a. In the still water, no obvious tendency was observed in the horizontal plane, as shown in Fig. 5b.

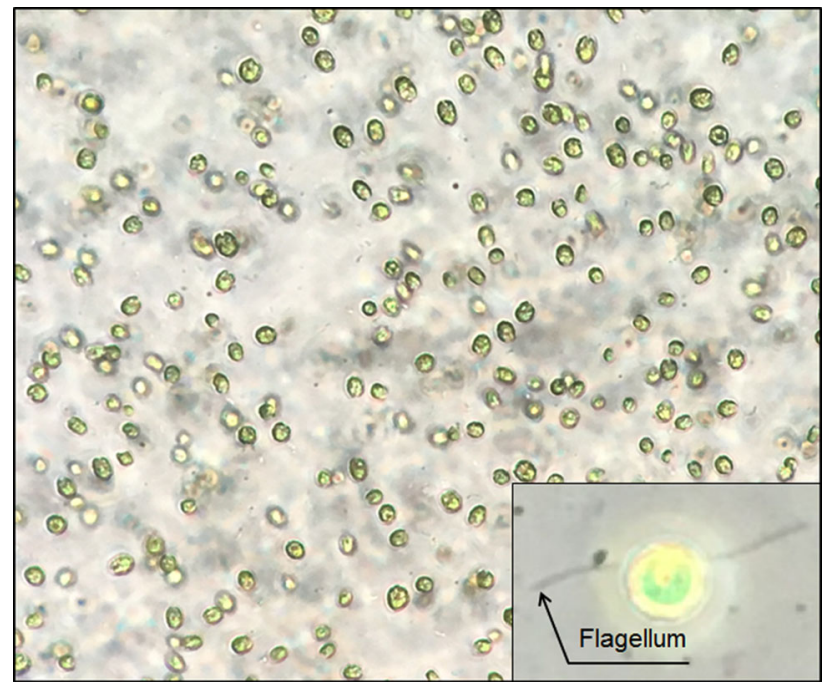

Fig. 4 C. reinhardtii under the microscope

\subsection{Flow in a microchannel}

Four flow conditions, adjusted by the discharge module of microfluidic system, were adopted in this experiment, as shown in Table 1. The flow pattern and cell swimming in the main channel were observed and analyzed at the observation position depicted in Fig. 1. The Reynolds number $(\operatorname{Re})$ in the main channel is defined as $\operatorname{Re}=\bar{u} L / v$, where $\bar{u}$ is the average flow velocity, $L(50 \mu \mathrm{m})$ is the depth of the microchannel and $v\left(0.877 \times 10^{-6} \mathrm{~m}^{2} / \mathrm{s}\right.$ at $\left.26^{\circ} \mathrm{C}\right)$ is the kinematic viscosity.

A laminar flow was observed in the channel when the potassium permanganate solution and ultrapure water were injected into the channel from the inlets $\mathrm{B}$ and $\mathrm{A}$, respectively. The flow patterns near the inlet B are shown in Fig. 6. The results showed that there was no vortex formation at the interface between the potassium permanganate solution and the ultrapure water, indicating the flow in the main channel was a low-Reynolds-number laminar flow.

The width-to-depth ratio of the main channel is 10 in present experiment. The velocity distribution in the cross section is shown in Fig. 7.

\subsection{Swimming velocity in low-Reynolds-number flow}

Figure 8 presents the probability density distribution of $U_{r}$, $V_{x}$ and $V_{y}$. The $U_{r}$ corresponding to the peak of probability distribution increases with the flow velocity, as shown Fig. 8a. The mean swimming velocity in the streamwise direction enhances as the velocity of ambient flow increases for the current flow conditions, as shown in Fig. 8b. The randomness of cell swimming still exists even for the case of moving water. The maximum swimming velocity in the $x$ direction in the moving water is larger than that in the still water. The average of $V_{x}$ is approximately $35 \mu \mathrm{m} / \mathrm{s}$ for conditions A1-4, which is slightly less than the average swimming speed in the still water. $V_{y}$ is subject to the Gaussian distribution with expectation value approximately equal to 0.0, as shown in Fig. 8c.

\subsection{Swimming direction in low-Reynolds-number flow}

The swimming direction of more than $80 \%$ cells is in the range of $90^{\circ} \pm 34^{\circ}$ for conditions A1-4, and the influence of cell randomness on cell migration becomes weak with the increasing flow velocity in the main channel, as shown in Table 2 and Fig. 9a. The swimming direction is approximately subject to Gaussian distribution, $A /(\sqrt{2 \pi} \sigma) \mathrm{e}^{-\left(\theta-\theta_{c}\right) /\left(2 \sigma^{2}\right)}$, where $\theta_{c}$ depends on flow little, 
Fig. 5 Swimming speed and direction of $C$. reinhardtii cells: a PDF of swimming speed and b distribution of swimming direction

Table 1 Experimental conditions

Fig. 6 Flow patterns in the main channel: a $\bar{u}=200 \mu \mathrm{m} / \mathrm{s}$, b $\bar{u}=2667 \mu \mathrm{m} / \mathrm{s}$ and c $\bar{u}=4667 \mu \mathrm{m} / \mathrm{s}$

Fig. 7 Velocity distribution: a velocity contours corresponding to condition A1, b velocity profile at $y=0$ for conditions A1-4 and c velocity profile at $z=0$ for conditions A1-4
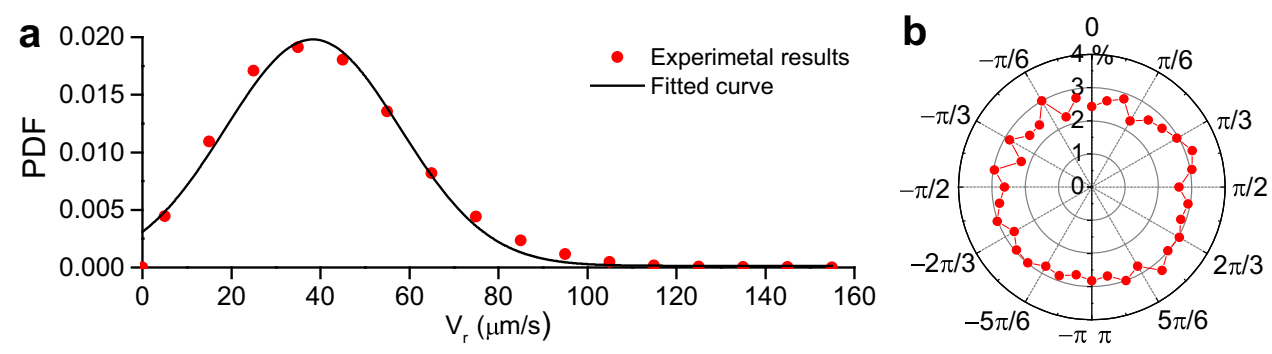

\begin{tabular}{llcl}
\hline Conditions number & Discharge $(\mathrm{nl} / \mathrm{min})$ & Velocity $(\mu \mathrm{m} / \mathrm{s})$ & Re \\
\hline A1 & 100 & 66.66 & $3.80 \mathrm{E}-03$ \\
A2 & 300 & 200.00 & $1.14 \mathrm{E}-02$ \\
A3 & 500 & 333.33 & $1.90 \mathrm{E}-02$ \\
A4 & 700 & 466.66 & $2.66 \mathrm{E}-02$ \\
\hline
\end{tabular}
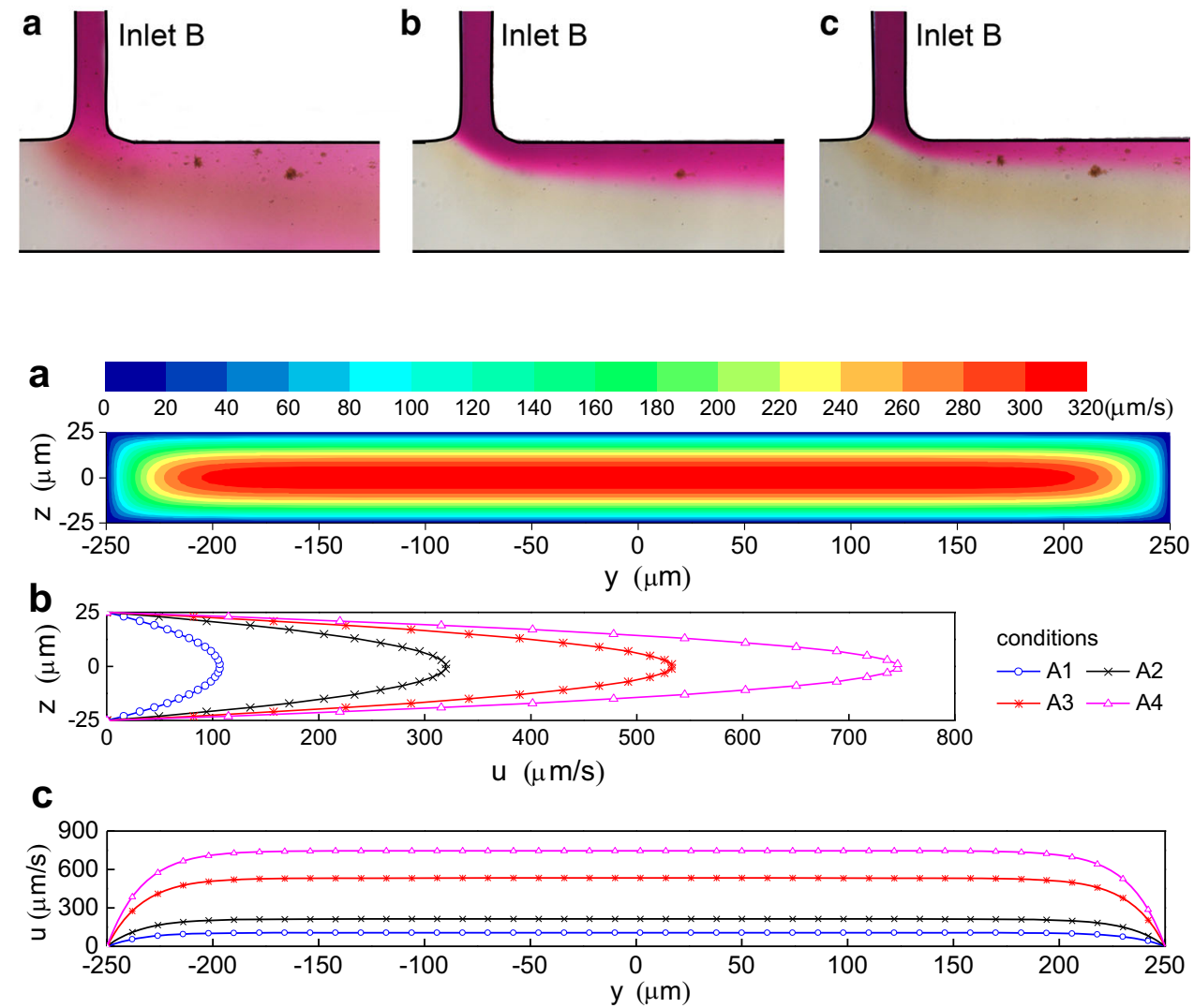

and $\sigma$ decreases with the increase of flow velocity, as shown in Fig. 9a, b. The migration of algal cells is influenced by the ambient flow, as shown in Fig. 9c. It is shown that $80 \%$ of the cells were aligned with the ambient flow when the flow velocity is greater than $333 \mu \mathrm{m} / \mathrm{s}$. $\sigma$ and the percent of swimming direction in the streamwise direction $\left(90^{\circ} \pm 3^{\circ}\right)$ can reach a stable status, when the flow velocity in the main channel increases up to $333 \mu \mathrm{m} / \mathrm{s}$.

\section{Discussion}

The measured mean speed of C. reinhardtii, based on 2407 cells, in the present work is approximately $41 \mu \mathrm{m} / \mathrm{s}$ for the still water. Sineshchekov et al. (2000) reported that the mean velocity of $C$. reinhardtii was 47.5 and $60 \mu \mathrm{m} / \mathrm{s}$ in the light-adapted and dark-adapted cultures for a red background illumination, respectively, as well as 36 and 

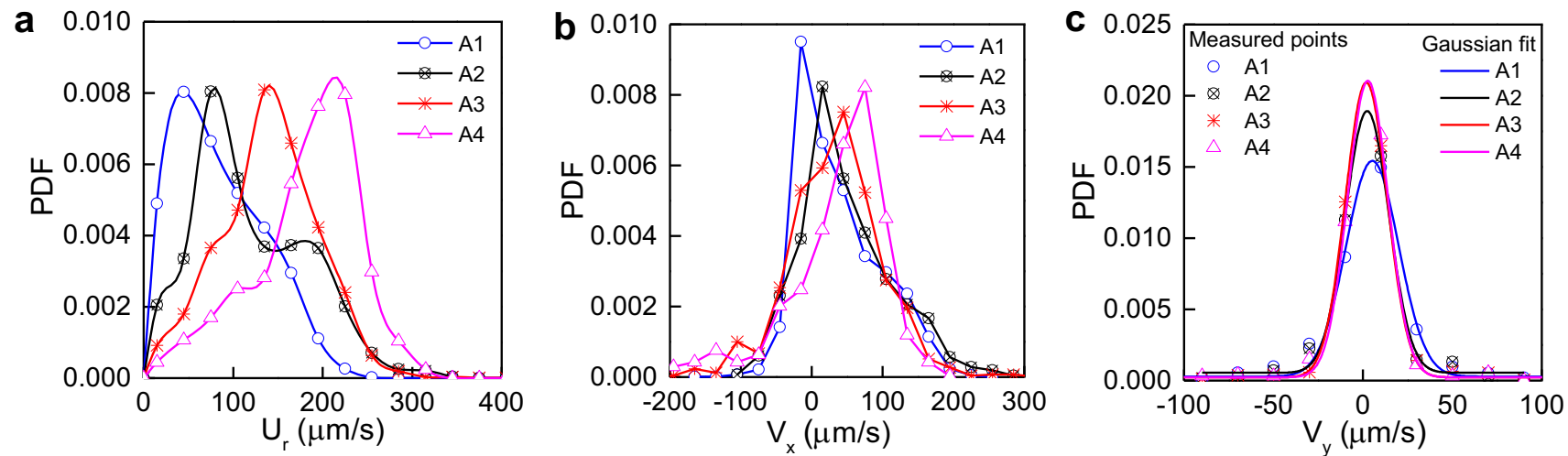

Fig. 8 PDF of $U_{r}, V_{x}$ and $V_{y}$ for conditions A1-4

Table 2 Swimming direction

\begin{tabular}{lll}
\hline Conditions number & Percent $(\%)$ & Swimming direction $\left({ }^{\circ}\right)$ \\
\hline A1 & 80 & $90^{\circ} \pm 34^{\circ}$ \\
A2 & 80 & $90^{\circ} \pm 14^{\circ}$ \\
A3 & 80 & $90^{\circ} \pm 3^{\circ}$ \\
A4 & 80 & $90^{\circ} \pm 3^{\circ}$ \\
\hline
\end{tabular}

$44 \mu \mathrm{m} / \mathrm{s}$ in the absence of red background illumination, respectively. The swimming speed in the present work is close to that in the absence of red illumination. The probability distribution measured in the present study is similar to that by Leptos et al. (2009). The mean speed, $41 \mu \mathrm{m} / \mathrm{s}$, observed in this work, is less than that (about $80 \mu \mathrm{m} / \mathrm{s}$ ) measured by Leptos et al. (2009). The difference is possibly caused by the differences of cell growth environment. The culture in the present work is SE medium, which is different from that (Tris-Acetate-Phosphate medium) used by Leptos et al. (2009). Furthermore, the mean swimming speed of $C$. nivalis and $H$. akashiwo, similar to $C$. reinhardtii in the shape and swimming behavior, is $55 \mu \mathrm{m} / \mathrm{s}$ (Vladimirov et al. 2004) and $40 \mu \mathrm{m} / \mathrm{s}$ (Bearon and Grünbaum 2008) in the still water, respectively.

The flow velocity is obtained by an analytical solution rather than a direct experimental measurement. In the present work, the focal plane is considered as a fixed plane with zero thickness, which may lead to a small position difference due to the existence of focal depth.

\section{Conclusions}

The swimming characteristics of a typical gyrotactic microorganism, C. reinhardtii, were studied for a lowReynolds-number flow, based on a microfluidic system. The measured swimming speed of $C$. reinhardtii for the still water in the present work, ranging from 0 to $160 \mu \mathrm{m} / \mathrm{s}$, is subject to Gaussian distribution. The swimming direction
Fig. 9 Swimming direction in various flow conditions: a PDF of swimming direction, b standard deviation $\sigma$ corresponding to the PDF and c percent of swimming direction
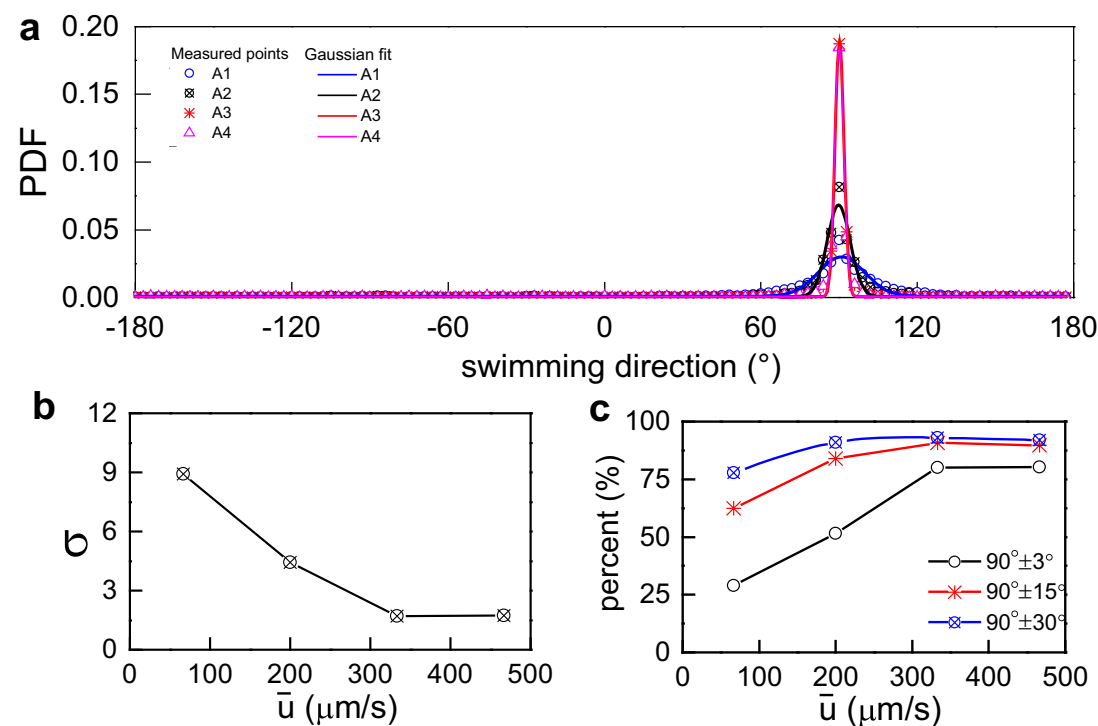
is still subject to Gaussian distribution for the still water. The ambient flow can influence the swimming behavior of cells, which results in a steeper variation of probability density distribution of swimming velocity in the cross-flow direction. It is also found that the maximum of swimming velocity aligned with the flow direction increases to some extent for the moving water. The streamwise mean swimming velocity in the ambient flow is slightly less than that in the still water. It is also shown that the swimming direction in the horizontal plane was dominated by cell randomness for the still water, and $80 \%$ of the cells were aligned with the ambient flow when the flow velocity exceeds $333 \mu \mathrm{m} / \mathrm{s}$. $\sigma$ and the percent of streamwise swimming direction $\left(90^{\circ} \pm 3^{\circ}\right)$ can reach a stable status, when the flow velocity in the main channel increases up to $333 \mu \mathrm{m} / \mathrm{s}$.

Acknowledgements This work is supported by the IWHR Research and Development Support Program(HY0145B402016 and HY0145B682017), and partly by the Independent Research Project of State Key Laboratory of Simulation and Regulation of Water Cycle in River Basin (2015RC01 and 2015ZY04).

\section{Compliance with ethical standards}

Conflict of interest The authors declare no conflict of interest.

\section{References}

Bearon RN, Grünbaum D (2008) From individual behaviour to population models: a case study using swimming algae. $\mathrm{J}$ Theor Biol 251(4):679-697

Croze OA, Sardina G, Ahmed M, Bees MA, Brandt L (2013) Dispersion of swimming algae in laminar and turbulent channel flows: consequences for photobioreactors. J R Soc Interface 10(81):20121041

Drescher K, Leptos KC, Tuval I, Ishikawa T, Pedley TJ, Goldstein RE (2009) Dancing volvox: hydrodynamic bound states of swimming algae. Phys Rev Lett 102(16):468101. doi:10.1103/ PhysRevLett.102.168101

Durham WM, Stocker R (2012) Thin phytoplankton layers: characteristics, mechanisms, and consequences. Annu Rev Mar Sci 4(1):177-207. doi:10.1146/annurev-marine-120710-100957

Durham WM, Kessler JO, Stocker R (2009) Disruption of vertical motility by shear triggers formation of thin phytoplankton layers. Science 323(5917):1067-1070. doi:10.11126/science.1167334

Durham WM, Climent E, Stocker R (2011) Gyrotaxis in a steady vortical flow. Phys Rev Lett 106(23):238102. doi:10.1103/ PhyRevLett.106.238102

Durham WM, Climent E, Barry M, Lillo FD, Boffetta G, Cencini M, Stocker R (2013) Turbulence drives microscale patches of motile phytoplankton. Nat Commun 4:2148. doi:10.1038/ncomms3148

Goldstein RE (2015) Green algae as model organisms for biological fluid dynamics. Annu Rev Fluid Mech 47(1):343-375. doi:10. 1146/annurev-fluid-010313-141426
Häder DP, Lebert M (1985) Real time computer-controlled tracking of motile microorganisms. Photochem Photobiol 42(5):509-514

Heisler J, Glibert PM, Burkholder JM, Anderson DM, Cochlan W, Dennison WC, Dortch Q, Gobler CJ, Heil CA, Humphries E, Lewitus A, Magnien R, Marshall HG, Sellner K, Stockwell DA, Stoecker DK, Suddleson M (2008) Eutrophication and harmful algal blooms: a scientific consensus. Harmful Algae 8(1):3-13. doi:10.1016/j.hal.2008.08.006

Hill NA, Häder DP (1997) A biased random walk model for the trajectories of swimming micro-organisms. J Theor Biol 186(4):503-526

Hwang Y, Pedley TJ (2014a) Bioconvection under uniform shear: linear stability analysis. J Fluid Mech 738(1):522-562. doi:10. 1017/jfm.2013.604

Hwang Y, Pedley TJ (2014b) Stability of downflowing gyrotactic microorganism suspensions in a two-dimensional vertical channel. J Fluid Mech 749:750-777. doi:10.1017/jfm.2014.251

Kessler JO (1985) Hydrodynamic focusing of motile algal cells. Nature 313:218-220

Leptos KC, Guasto JS, Gollub JP, Pesci AI, Goldstein RE (2009) Dynamics of enhanced tracer diffusion in suspensions of swimming eukaryotic microorganisms. Phys Rev Lett 103(19):198103. doi:10.1103/PhyRevLett.103.198103

Lillo FD, Cencini M, Durham WM, Barry M, Stocker R, Climent E, Boffetta G (2014) Turbulent fluid acceleration generates clusters of gyrotactic microorganisms. Phys Rev Lett 112(4):044502

Marcos, Stocker R (2006) Microorganisms in vortices: a microfluidic setup. Limnol Oceanogr Methods 4(10):392-398

Martin M, Barzyk A, Bertin E, Peyla P, Rafai S (2016) Photofocusing: light and flow of phototactic microswimmer suspension. Phys Rev E 93(5):051101

Pedley TJ, Kessler JO (1990) A new continuum model for suspensions of gyrotactic micro-organisms. J Fluid Mech 212:155-182

Pedley TJ, Hill NA, Kessler JO (1988) The growth of bioconvection patterns in a uniform suspension of gyrotactic micro-organisms. J Fluid Mech 195:223-237

Rusconi R, Guasto JS, Stocker R (2014) Bacterial transport suppressed by fluid shear. Nat Phys 10(3):212-217. doi:10. 1038/NPHYS2883

Sengupta A, Carrara F, Stocker R (2017) Phytoplankton can actively diversify their migration strategy in response to turbulent cues. Nature 543(7646):555-558. doi:10.1038/nature21415

Shah RK, London AL (1978) Laminar flow forced convection in ducts. Academic Press, New York

Sineshchekov O, Lebert M, Häder DP (2000) Effects of light on gravitaxis and velocity in Chlamydomonas reinhardtii. J Plant Physiol 157(3):247-254

Son K, Brumley DR, Stocker R (2015) Live from under the lens: exploring microbial motility with dynamic imaging and microfluidics. Nat Rev Microbiol 13(12):761-775. doi:10.1038/ nrmicro3567

Vladimirov VA, Denissenko PV, Pedley TJ, Wu M, Moskalev IS (2000) Algal motility measured by a laser-based tracking method. Mar Freshw Res 51(6):589-600

Vladimirov VA, Wu MSC, Pedley TJ, Denissenko PV, Zakhidova SG (2004) Measurement of cell velocity distributions in populations of motile algae. J Exp Biol 207(Pt7):1203-1216. doi:10.1242/ jeb.00881

White FM (2005) Viscous fluid flow, 3rd edn. McGraw-Hill, Singapore 